\title{
The Effectiveness of a Short Cognitive Behavioural Training Course on Awareness, Knowledge, and Transferability of Competencies in Clinical Practice
}

\author{
Andrew Edward Paul MITCHELL, \\ PhD, RNMH, CPsychol, FHEA, is a Senior Lecturer, Mental Health, University of \\ Chester, Chester, UK.
}

\begin{abstract}
PURPOSE: In this study we investigated the effects of training on knowledge acquisition and core competencies in CBT.
\end{abstract}

DESIGN AND METHODS: Forty three students attended 15 half day, weekly sessions and were tested with the Cognitive Therapy Awareness Scale (CTAS) at weeks 1 and 15 in a before and after study. The students case studies were assessed with competencies items 7-12 on the Cognitive Rating Scale - Revised (CTS-R).

FINDINGS: Improvements in the CTAS were modest. Key competencies on the CTS$\mathrm{R}$ subscales at week 15 were also observed.

PRACTICE IMPLICATIONS: CBT knowledge acquisition might improve patients' outcomes through impact on competencies.

\section{Search terms}

Cognitive and Behavioural Therapy, Education, Training, competencies, case studies

\section{Introduction}

The interest in core competencies in psychotherapy has soared and the particular focus of cognitive and behavioural therapies has led to unprecedented interest in practice skills (Keegan, 2012). There is also a patient preference for cognitive behavioural therapies rather than pharmacotherapies, as the latter has known side effects such as weight gain (Gøtzsche, 2014). Cognitive behavioural therapies have been shown to work for a variety of emotional disorders, and a recent meta-analysis has confirmed the efficacy (Hofmann, Asnaani, Vonk, Sawyer, \& Fang, 2012). Prior to this study, there has been studies exploring training effects in Cognitive and 
Behavioural Therapy (CBT), utilizing written assignment assessment and exams, but these have not necessarily been linked with competencies (Keen \& Freeston, 2008; Rakovshik, \& McManus, 2010). However, the authors suggested that further investigation was required into the use of written assignments and core competencies assessment.

There has been increased interest in evidenced based practice and competencies in psychotherapy (Roth, 2014). The standard for evidenced based practice suggests that the procedure must be defined in a manual and found efficacious in at least two randomized controlled clinical trials (Rakovshik \& McManus, 2010). There has also been a growing demand for training and short training courses, which focus on cognitive behavioural approaches, looking at focused CBT interventions and problem specific evidenced based techniques for specific disorders (Roth \& Pilling, 2007). Key competencies were recommended for delivering these techniques and implemented as a result. The main content areas include: generic competencies in psychological treatments, basic cognitive-behavioural competencies, specific cognitive and behavioural techniques, problem-specific CBT skills, and metacompetencies to adapt CBT to the needs of each client.

Myles and Milne (2004) evaluated the impact of a 12 week shared learning programme in CBT. The findings indicated that a short training programme was effective in knowledge and skills acquisition and generalization to work environment. The authors indicate a limitation in that no direct observation of behaviour change was used following training, such as submission of assessment designed to assess application to clinical practice. Westbrook, Sedgwick-Taylor, Bennett-Levy, Butler, 
and McManus (2008) evaluated the impact of 10 day training course on trainees' satisfaction, clinical skill and patient outcomes. The authors report improvements in CBT competencies rated by the Cognitive Therapy Scale (CTS; Young \& Beck, 1980). Although a patient outcome assessment was used to investigate benefits to patients, the analysis did not allow for correlational analysis between CTS scores and patient outcome assessment. Fairburn and Cooper (2011) looked at current methodological aspects to assessing competence, therapy quality and therapist training and provide a review of the current status on methods used to assess therapist competence in application to practice. The main methods involved evaluation of patient outcome and evaluation of competencies rated against CTS. Overall, studies evaluating the impact of training rarely look at generalisation of knowledge acquisition in clinical practice. The present study attempts to address this issue by using clinical case studies to assess the application in clinical practice by assessing the competencies rated against key skills on CTS.

\section{Assessing competence}

There is a need to demonstrate that short training courses are effective in order to ensure good use of scarce staff resources (Milne, Baker, Blackburn, James, \& Reichelt, 1999). There is good reason to assess practitioner competencies in CBT as delivering interventions in a competent manner is seen to enhance treatment fidelity (Di Giulio, 2010). The practitioners' competence can be described as the extent to which the practitioner has the knowledge and skills required to deliver a treatment to achieve an effect (Fairburn \& Cooper, 2011). The students undertaking CBT training need to bring together knowledge, skills and attitudes into their practice (Roth \& Pilling, 2007). Key competencies have been organised into five domains: generic 
competencies such as agenda setting, ability to plan and review practice assignments; basic cognitive and behavioural competencies, such as knowledge of basic principles of CBT and rationale for treatment and ability to structure sessions; specific CBT techniques including cognitive techniques such as guided discovery and using thought record; behavioural techniques such as exposure procedures, activity monitoring and scheduling; problem-specific CBT skills such as treating specific conditions including depression and anxiety disorders; and metacompetencies including the ability to use clinical judgement when implementing a course of CBT treatment and capacity to implement CBT in a manner cognizant with the treatment ethos (Roth \& Pilling, 2007). The latter is beyond the scope of the present study due to the educational level of the short course.

The present study evaluated the impact of a short training course on knowledge and transfer into practice. It was hypothesized that there would be a significant increase in pre to post training Cognitive Therapy Awareness Scale (CTAS; Myles \& Milne, 2004). Also, that there would be a significant correlation between post CTAS and Cognitive Rating Scale - Revised (CTS-R; Blackburn et al., 2001) on competencies $7-12$. As far as the author is aware, this is the first study that combines the case study assessment with the CTS-R scale to assess impact of training. Cognitive and behavioural training consists of educational and clinical practice components which, are integral to practitioners' educational experience. These practitioners are a valuable resource for the health system to meet future workload demands. The training of non-specialist CBT practitioners to increase awareness and knowledge of cognitive and behavioural approaches is based on the premise that increased awareness and knowledge will lead to increased competence in implementing CBT 
skills in practice (Strunk, Brotman, DeRubeis, \& Hollon, 2010). However, limited evidence exists on the effectiveness of such training techniques and the transfer of knowledge and skills into the practice settings. To meet these requirements students' competencies must be evaluated. Therefore, it is crucially important to evaluate training, competencies in cognitive and behavioural training and its transfer into the practice setting.

\section{Methods}

Aims

The research aims originated from the intention to perform future work on the impact of training in practice. The aim was to investigate the impact of a CBT module, 15 half-day sessions over 15 weeks, at level 6 (e.g. equivalent to studying at the level of Bachelor's degrees with honours, graduate certificates and graduate diplomas) on the awareness and knowledge of cognitive behavioural therapy. Also, to what extent students incorporate these competencies into their clinical practice. The objectives were to:

- evaluate participant knowledge and awareness of CBT before and after the training course

- assess the case study report as a tool to assess skills in practice

\section{Participants}

The participants were 43 non-CBT practitioners. Participants were students at a University in the Northwest of England in health and social care programmes. The inclusion criteria included being registered on the course and completing all course work requirements of the module. Exclusion criteria includes those students not undertaking coursework or being unavailable to completed both week 1 and week 15 
study questionnaires. Students were recruited by the distribution of an invite pack containing a participant information sheet and consent form. The invites were given to all students attending the CBT module. The flow of participants through each stage of the study was as follows: enrollment stage, 46 attendees on the CBT modules over two semesters, $N=43$ volunteered to participate and $n=3$ people were unavailable on the commencement date due to work commitments or sickness. Allocation to intervention stage, $N=43$. Follow up stage, $N=43$ students went on to complete the training and had to complete a minimum of $80 \%$ attendance to complete the module. The sample consisted of 28 female (65.1\%) and 15 male (34.9\%) participants. There were 7 different professional groups represented; 35 Mental Health Nurses, 3 Counselors, 2 Health and Wellbeing Practitioners, 1 Occupational Therapist, 1 Social Worker and 1 Physiotherapist. The sample demographics are in keeping with previous module attendees (see Table 1).

\section{Insert Table 1 in this location}

A post hoc power analysis was conducted using the software package, GPower (Faul, Erdfelder, Lang, \& Buchner, 2007). The sample size of 43 was used for the statistical power analyses. The recommended effect sizes used for Wilcoxon matched pair were as follows: small $(f=.15)$, medium $(f=.33)$, and large $(f=.47)$ (Cohen, 1977). The alpha level used for this analysis was $p<.05$. The post hoc analyses revealed the statistical power for this study was .16 for detecting a small effect, .54 for detecting a moderate effect, .84 for detecting a large effect size. Thus, there was more than adequate power (i.e., power .80) at the large effect size level, but less than adequate statistical power at the small to moderate effect size levels. 


\section{Measures}

Awareness and knowledge of CBT

The 'Cognitive Therapy Awareness Scale' (CTAS; Myles \& Milne, 2004) has 40 true / false statements composed into ten key questions. The four true / false statements in each question result in a score between $0-4$. The sum of each question was calculated at both time intervals for Wilcoxon signed-rank test comparison. The post CTAS scores were used in the Spearman rho correlations. The scale was used to measure changes in awareness and knowledge of cognitive and behavioural therapy as a pre- and post-measure. Participants completed the measure on pre training session 1 (Week 1) and post training session 15 (week 15). The maximum score on the CTAS is 40.

\section{Written case study}

The case studies were assessed against the Cognitive Therapy Scale-Revised (CTS-R; Blackburn et al., 2001), by the course leader, using the structured and objective anchor points to limit marker bias. The rationale for the use of items $7-12$ only was twofold; these reflected the CBT specific competencies rather than the more generic psychotherapeutic competencies reflected in items $1-6$. Furthermore, items $7-12$ could be assessed by evidence presented in the case studies which assessed four key aspects to CBT practice (1) assessment (2) formulation (3) intervention and (4) evaluation. These key aspects to the case study provided content on which to assess competencies items 7-12 on the CTS-R (Blackburn et al., 2001). 


\section{Rating of competencies and transfer to practice}

Cognitive Therapy Scale-Revised (CTS-R) was used to measure competencies in cognitive and behavioural therapy (Blackburn et al., 2001). The CTS-R can be used to rate student's overall performance or to focus on specific skills (Dryden \& Branch, 2012). The present study focuses on the CTS-R for specific skills. There was no attempt to produce an overall score to judge overall competency in CBT. Instead individual items were scored to focus on individual competencies in a specific area. Items 1- 6 included agenda setting and adherence, feedback, collaboration, pacing and efficient use of time, interpersonal effectiveness and eliciting appropriate emotional expression. Items $1-6$ were not assessed in the case studies due to difficulty in assessing such competencies from the case study assessments. Items 7

- 12 assess the ability to elicit key cognitions, ability to elicit and plan behaviours, ability to use guided discovery and conceptual integration, the application of change methods and ability to incorporate and use homework settings. The items are carefully defined and rated on a six point competency scale. This unipolar Likert Scale extends from 'incompetent' (level 0) to 'expert' (level 5).

The short training course

The course consists of teaching sessions in assessment, formulation, and intervention, and relapse prevention. The content of the course sought to provide participants with a basic theoretical understanding of the principles and underpinning cognitive and behavioural interventions. The material was delivered using a diversity of learning methods including presentations, group work demonstrations and experiential role-plays. The short training course concentrates on fundamental CBT 
competencies in the application of CBT in clinical practice: assessment, goal setting, structuring sessions, formulation, Socratic methods; identifying and challenging automatic thoughts, identifying and re-evaluating dysfunctional assumptions; behavioural experiments, relapse prevention. The students also received evidencebased disorder-specific protocols. Sessions covered: an overview of depressive disorders and risk associated with mood disorders, anxiety disorders; panic, phobia and social anxiety disorders (see Table 2).

\section{Insert Table 2 in this location}

Each training session included a mixture of didactic presentations, video demonstrations of sessional content, case studies, interactive exercises and discussions. Session themes included the importance of collaboration, socialisation into CBT principles, methods for debriefing homework, emphasis on Socratic methods to emphasize client learning. During the course, practitioners are expected to be working in clinical practice and see patients with a range of mental health problems in their normal work settings.

The study design

Ethical approval for the study was sought and granted from the institutions ethical committee. All participants received written information and signed consent forms prior to completing outcome scales and made aware that their participation could be withdrawn at any time. The only adverse effects observed in the study was the anticipated anxiety by participants on the prospects of completing a questionnaire testing their knowledge of $\mathrm{CBT}$. Otherwise no adverse effects were observed in the study. 
The design was a quasi-experimental before and after study. This was chosen due to the unknown impact of CBT training on knowledge and the ability of the proposed case study as a way to assess competencies. The independent variable, attending the module, was expected to affect awareness and knowledge, which was measured by the Cognitive Therapy Awareness Scale (CTAS; Myles \& Milne, 2004). The marker was blind to the CTAS scores when rating the CTS-R. It was predicted that a 15 week training course would lead to improvements in knowledge and awareness of CBT. Participants were aware that awareness and knowledge was being tested but no firm link with competencies was highlighted. The students understood that case studies would be assessed with the CTS-R without the firm link being emphasised. It is recognised that participants could have guessed the aim of the study but no one raised this during the initial discussion or debriefing. The within-subjects design was utilized to reduce the variance associated with individual differences. The secondary dependent measures were competency items 7 - 12 on the Cognitive Therapy Rating Scale - Revised (CTS-R; Blackburn, James, Milne, \& Reichelt, 2001). It was predicted that the post CTAS scores would be correlated with a number of competency scores on CTS-R.

\section{Procedure}

At the start of the module, the purpose of the study was explained and informed consent was sought from each participant. Participants were advised that information they provided would be used for research and publications. The participants were told the type of information that would be obtained and the nature of the commitment. Confidentiality pledge was made and the researcher clearly indicated 
that participation was strictly voluntary and no penalties or losses will be incurred by non-participation.

Students completed the questionnaires and academic assignments according to the following schedule:

a) Following written informed consent at session 1, participants completed the CTAS. The questionnaire was completed before any formal training in CBT took place. At session 15, the same participant group was again administered CTAS. This was followed by a debriefing session.

b) The knowledge and skills delivery was according to the pre-set module aims and weekly sessional content.

c) The academic assignment was based on a single case study and submitted on session 15 and rated on the CTS-R by the author.

Students received written formative feedback as well as a numerical mark. Case studies were double marked, blind as to candidate, with a mark of $40 \%$ or above constituting a pass. To optimise the reliability and validity of the CTS-R coding, a list of written statements referring to each component was available at the time of scoring, indicating a value to be scored. This was done in the absence of the CTAS mark awarded. No further reliability checks were conducted. The CTAS questionnaires were conducted under exam like conditions and marked by the first author. The author marked each case study according to the academic level 6 marking criteria and assessed each case study against the CTS-R items $6-12$ as these assessed specific Cognitive-Behavioural Techniques (see Rating of competencies and transfer to practice section). 


\section{Data analysis}

The present study reports summary scores from the CTAS (Cognitive Therapy Awareness Scale) and the subscales of the CTS-R (Cognitive Therapy ScaleRevised). Statistical advice was sought from a statistician and APA guidelines adhered to for data presentation. The sum of the pre- and post-test scores for each participant were calculated and a test of difference was performed to determine if the responses changed from pre to post in a statistically significant manner. Significance was set at $95 \%$ where $p<.05$ for the test of difference. Normality was determined with the Shapiro-Wilk test.

\section{Results}

A Wilcoxon signed-rank test was selected to test for differences on the CTAS questionnaire between session 1 and session 15 . There was a significant increase in CTAS scores $(N=43)$ between 1 st session $($ Median $=28$, Interquartile range $(I Q R)=$ 6 ) and 15th session (Median $=30, I Q R$ 6) showing an increase in knowledge and awareness in CBT $(Z=-2.07, d f=42, p=.04)$. As the internal consistency (i.e. general agreement between multiple items that make up a composite score) of the CTAS is high, the improvements probably reflect the effectiveness of the training module. A Wilcoxon signed-rank test was conducted on pre to post CTAS items. Four out of the ten key questions indicated a significant positive higher median posttest score. Specifically, questions reflecting knowledge of levels of cognition such as automatic thoughts and schemas (item 1) and knowledge of cognitive and behavioural aspects, such as examining the evidence for beliefs and activity scheduling (item 2), both indicated that the median post-test scores (Median $=3.5$, $I R Q=1$ and Median $=3, I R Q=2$ respectively) were statistically significantly higher 
than the median pre-test scores (Median $=3, I Q R=2$ and Median $=2, I Q R=2$ respectively) and Wilcoxon test scores $(Z=-2.39, p=.02$ and $Z=-4.40, p=<.001$ respectively). The increase in awareness also reflected an increase in reported application to practice questions using cognitive techniques, such as cognitive errors and exploring cognitive distortions (item 7), and using behavioural techniques, such as activity scheduling and recording mastery and pleasure (item 9). Again, both indicate that the median post-test scores (Median $=4, \mathrm{IRQ}=1$ and Median $=4, \mathrm{IRQ}$ $=0$ respectively) were statistically significantly higher than the median pre-test scores $($ Median $=2, I Q R=1$ and Median $=4, I Q R=1$ respectively) and Wilcoxon test scores $(Z=-3.61, p=<.001$ and $Z=-3.37, p=<.001$ respectively). Table 3 presents Wilcoxon signed rank test scores on CTAS items pre and post training.

\section{Insert Table 3 in this location}

A series of Spearman rank-order correlations were conducted in order to determine if there were any relationships between post CTAS and CTS-R competency components. A one-tailed test of significance indicated that there was a significant positive relationship between post CTAS and CTS-R component $7\left(r_{s}=.29, p=.03\right.$, $d f=42$ ). This means that the practitioner's cognitive awareness was significantly correlated with the ability to elicit key cognitions. This component assesses the general work done with eliciting key cognitions. The key features of 'Eliciting Key Cognitions' is outlined in the CTS-R Rating Scale as follows: "To help the patient gain access to his/her cognitions (thoughts, assumptions and beliefs) and to understand the relationship between these and their distressing emotions. This can be done through the use of questioning, diaries and monitoring procedures" (Blackburn et al., 2001 p9). There was a second significant positive correlation between CTAS and 
CTS-R component $9\left(r_{s}=.35, p=.01, d f=42\right)$. This means that the practitioner's cognitive awareness was significantly correlated with their ability to use guided discovery. This component assesses the effective use of questioning techniques by the practitioner to assist the patient to gain new perspectives for himself / herself. The patient is helped to develop a range of perspectives regarding his / her experience. A third significant positive correlation was between CTAS and CTS-R component $10\left(r_{s}=.35, p=.01, d f=42\right)$. This component assesses the practitioner's ability to conceptually integrate and propose a psychological understanding of the patient's problem. This means that the practitioner's cognitive awareness was significantly correlated with the ability to understand a theory based on psychological conceptualization, to guide the patient's treatment plan. There was a fourth significant positive correlation between CTAS and CTS-R component $11\left(r_{s}=.27, p\right.$ $=.04, d f=42)$. This component assesses the practitioner's ability to appropriately use the application and knowledge of change strategies. This means that the practitioner's cognitive awareness was significantly correlated with ability to apply change strategies within practice.

Table 4 presents the Spearman's rho correlation coefficients between the post scores on the CTAS and the competencies scores observed at week 15.

\section{Insert Table 4 in this location}

\section{Discussion}

It was hypothesized that there would be a significant increase in pre to post training CTAS and there would be a significant correlation between post CTAS and CTS-R on competencies $7-12$. The key findings in the present study suggest that the 
cognitive and behavioural training course showed a significant increase in knowledge and understanding of cognitive and behavioural approaches. The results of this study demonstrated that by session 15 the participants' cognitive awareness was significantly correlated with a number of competencies; ability to elicit key cognitions, ability to use guided discovery, ability to conceptually integrate therapeutic strategies within practice, and ability to apply change methods within practice. The CTS-R competencies 8 and 12, namely eliciting behaviours and homework setting showed non-significant correlations. However, the scores for these competencies on CTS-R revealed reasonable levels of competence but the CTAS questions were broader rather than specifically assessing these two areas. As a consequence, the researcher cannot exclude this was due in part to the broader rather than specific questions assessing components 8 and 12 .

The post training CTAS scores showed the predicted increase, with items 1, 2, 7 and 9 showing a significant increase. CTAS items 5 and 10 did not reach the conventional level of significance at $p<.05$. It was however, approaching significance at $p=.06$ and these items may have reached significance given an increase in sample size. Items 3, 4, 6 and 8 showed a non-significant increase. However, the initial scores for these items revealed reasonable levels of knowledge present at pre training assessment. As a consequence, the increase from pre - post was non-significant due to the higher baseline levels on these items. A possible explanation for this is that student's background in mental health was already sufficient to address some knowledge questions on the CTAS. 
The participants' gains in awareness and knowledge are broadly consistent with studies in the literature for short courses in CBT (Milne et al., 1999; Myles \& Milne, 2004). The present findings support evidence that short training courses increase awareness and knowledge in students. The present study also extends these findings by attempting to show increased knowledge and transfer of key competencies to clinical practice. Unlike the present study, Milne et al. (1999) and Myles and Milne (2004) did not incorporate a measure to assess generalization to practice based competencies in CBT. The present study differed in its ability to demonstrate, to some extent, the transferability of knowledge into practice. The findings of transferability to practice are broadly consistent with studies incorporating transferability measures (Westbrook et al., 2008). However, the present study did differ in that it did not incorporate a direct measure to assess benefit to patients as in the later mentioned study. It is also recognised that other studies have shown that training has limited effect on competencies (Bennett-Levy, McManus, Westling, \& Fennell, 2009).

This contrasts with studies such as Simons et al. (2010) and Westbrook et al. (2008) that indicate increased effects of training on competencies being applied in practice. The present study did show that a focus on cognitive and behavioural skills, such as knowledge of levels of cognition, and behavioural aspects, such as activity scheduling, are important educational components. These components seem to increase self-reported application in practice with reported ability to understand the use of cognitive techniques, such as cognitive errors and exploring cognitive distortions, and ability to understand the use of behavioural techniques, such as activity scheduling and recording mastery and pleasure. 
The positive change in the practice application questions on the CTAS seem to reflect the reported competencies assessed by the CTS-R in the case studies looking at the application of these very skills in the students' practice setting during the short training module. The key competencies in the training reflected the main content delivered and include: assessment, how to implement the CBT model, approaches to unhelpful thinking, approaches to overcoming unhelpful behaviour and evidence base for CBT.

\section{Limitations}

The major constraint of this study was the fact that there was no control group so we do not have any scores for those not going through the course. The second main limitation is that the Cognitive Therapy Scale (CTS-R) ratings were based only on a review of written case studies, without the comparison, of an audio or video tape analysis. This had a limitation in that it only allowed for self-reports of skills by the practitioners and their ability to describe the practice components. The lack of a formal reliability check on rating the CTS-R does not allow for the possibility of bias to be excluded from results interpretation. Although the results suggest a positive correlation between post CTAS and CTS-R, the interpretation, that CTAS (knowledge and awareness) is positively correlated with CTS-R (competencies), is based on post CTAS and post CTS-R ratings. Ideally, in a future study, CTS-R (competencies ratings) could be assessed at the beginning and measured again at the end of the module as with the CTAS (knowledge and awareness). Therefore, caution should be taken when generalizing these results to similar short training courses. Assuming that future studies address the limitations then generalizability should be possible. 


\section{Conclusions}

The results of this study suggest that the participants significantly improved in knowledge and awareness, which was significantly correlated with a number of competencies, by the end of the module. The utilization of assessed case studies, which can be conducted without resource workload capacity issues required for direct observation, could be usefully incorporated with other methods in future research investigating cognitive and behavioural competencies. In conclusion, this study contributes to the issue of transfer of CBT knowledge and skills into practice. Further evaluation at 12 months post training, with assessment of patient outcome, would be useful to identify the extent and enduring changes in practice competencies.

\section{Implications for Nursing Practice}

Traditional measures of competencies require direct observation of clinical practice or audio taped sessions which require substantial staffing resources and ethical obstacles, especially for post registration short training courses. Scales that assess competencies in practice are increasing but seem to require observation of clinical practice rather than via self- reported case studies. While these direct measures provide a clear and important measure of competencies, they have limitations in that direct observation by an assessor is required when practitioner and client contact is being conducted or substantial time required to listen to audio taped sessions after the taped session. It would be good to see competency scales that can be used in conjunction with traditional based written case study assessment, which could be used across practice settings to assess short training courses. 
The present study suggests that even a short training course in CBT can enable mental health nurses, as well as other health professionals working in mental health, to apply the concepts learnt during a short course in CBT into their practice settings. However, training must also have a measurable effect on competencies in practice, if it is to be reflected in patient outcome. One obvious way to increase competencies in $\mathrm{CBT}$ is to utilize experienced practitioners. Advanced practitioner nurses working in environments where CBT is used are in an ideal situation to bring about training. Advanced practitioner nurses should consider the application of CBT training for staff in their practice setting and look at how to successfully integrate competency based assessment.

The next stage of research would be to measure and assess competencies that lead directly to improved patient outcome. Roth, Pilling, and Turner (2010) suggest that successful criterion for measuring impact of skills training should be patient outcome. A further refinement to future research would need to evaluate the practitioners' competencies by assessing the impact these have on patients outcomes in clinical practice.

\section{Acknowledgements}

This research received no specific grant from any funding agency in the public, commercial or not-for-profit sectors.

\section{References}

Bennett-Levy, J., McManus, F., Westling, B., \& Fennell, M.J.V. (2009). Acquiring and refining CBT skills and competencies: Which training methods are perceived to be most effective? Behavioural and Cognitive Psychotherapy, 37(5), 571-583. doi:org/10.1017/S1352465809990270 
Blackburn, I.M., James, I.A., Milne, D.L., \& Reichelt, F.K. (2001). The revised cognitive therapy scale (CTS-R): psychometric properties. Behavioural and Cognitive Psychotherapy, 29(4), 431-447. doi:org/10.1017/S1352465801004040

Cohen, J. (1977). Statistical power analysis for the behavioral sciencies. Hillsdale, NJ: Lawrence Erlbaum Associates.

Di Giulio, G. (2010). Therapist, client factors, and efficacy in cognitive behavioural therapy: A meta-analytic exploration of factors that contribute to positive outcome. Ottawa: University of Ottawa.

Dryden, W., \& Branch, R. (2012). The CBT Handbook. London: SAGE

Fairburn, C. G., \& Cooper, Z. (2011). Therapist competence, therapy quality, and therapist training. Behaviour Research and Therapy, 49(6-7), 373-378. doi:10.1016/j.brat.2011.03.005

Faul, F., Erdfelder, E., Lang, A.G., \& Buchner, A. (2007). GPower 3: A flexible statistical power analysis program for the social, behavioral, and biomedical sciences. Behavior Research Methods, 39(2), 175-191. doi:org/10.3758/BF03193146

Gøtzsche, P.C. (2014). Why I think antidepressants cause more harm than good. Lancet Psychiatry, 1(2):104-6. doi:org/10.1016/S2215-0366(14)70280-9

Hofmann, S.G., Asnaani, A., Vonk, I.J.J., Sawyer, A.T., \& Fang A. (2012). A review of meta-analyses of cognitive behavioral therapies. Cognitive Therapy and Research, 36(5), 427-440. doi:org/10.1007/s10608-012-9476-1

Keegan, E. (2012). Foreword. In C. F. Newman (Ed.), Core competencies in cognitive-behavioral therapy: Becoming a highly effective and competent cognitivebehavioral therapist (core competencies in psychotherapy series) (pp. xvii - xx). London: Routledge. 
Keen, A.J.A., \& Freeston, M.H. (2008). Assessing competence in cognitivebehavioural therapy. British Journal of Psychiatry, 193(1), 60-64. doi:org/10.1192/bjp.bp.107.038588

Milne, D. L., Baker, C., Blackburn, I. M., James, I., \& Reichelt, K. (1999). Effectiveness of cognitive therapy training. Journal of Behavior Therapy \& Experimental Psychiatry, 30(2), 81 -92. doi:org/10.1016/S0005-7916(99)00011-7

Myles, P.J., \& Milne, D.L. (2004). Outcome Evaluation of a brief shared learning programme in cognitive and behavioural therapy. Behavioural and Cognitive Psychotherapy, 32(2), 177-188. doi:org/10.1017/S1352465804001183

Rakovshik, S.G., \& McManus, F. (2010). Establishing evidence-based training in cognitive behavioral therapy: A review of current empirical findings and theoretical guidance. Clinical Psychology Review, 30(5), 496-516. doi:org/10.1016/j.cpr.2010.03.004

Roth, A.D. (2014). Are competence frameworks fit for practice? Examining the validity of competence frameworks for CBT, psychodynamic, and humanistic therapies. Psychotherapy Research, 25(4), 460-472 doi:org/10.1080/10503307.2014.906763

Roth, A.D., \& Pilling, S. (2007). The competencies required to deliver effective cognitive and behavioural therapy for people with depression and with anxiety disorders. London, Department of Health

Roth, A.D., Pilling, S., \& Turner, J. (2010). Therapist training and supervision in clinical trials: implications for clinical practice. Behavioural and Cognitive Psychotherapy, 38(3), 291 - 302. doi:org/10.1017/S1352465810000068

Simons, A.D., Padesky, C.A., Montemarano, J., Lewis, C.C., Murakami, J., Lamb, K., DeVinney, S., Reid, M., Smith, D.A., \& Beck, A.T. (2010). Training and dissemination of cognitive behavior therapy for depression in adults: A preliminary examination of 
therapist competence and client outcomes. Journal of Consulting and Clinical Psychology, 78(5), 751-756. doi:org/10.1037/a0020569

Strunk, D.R., Brotman, M.A., DeRubeis, R.J., \& Hollon, S.D. (2010). Therapist competence in cognitive therapy for depression: predicting subsequent symptom change. Journal of consulting and clinical psychology, 78(3), 429-437. doi.org/10.1037/a0019631

Westbrook, D., Sedgwick-Taylor, A., Bennett-Levy, J., Butler, G., \& McManus, F. (2008). A Pilot Evaluation of a Brief CBT Training Course: Impact on Trainees' Satisfaction, Clinical Skills and Patient Outcomes. Behavioural and Cognitive Psychotherapy, 36(5), 569-579. doi:org/10.1017/S1352465808004608

Young, J.E., \& Beck, A.T. (1980). Cognitive Therapy Scale manual. Philadelphia PA: Center for Cognitive Therapy, University of Pennsylvania. 
Table 1: Demographic characteristics

Variable

Age (years)

Gender

Male

Female

Professional groups

Mental Health Nurses

Counsellors

Health and Wellbeing Practitioners

Occupational Therapist

Social Worker

Physiotherapist
$M(S D) / n$

$38.2(9.8)$

100

15

28

34.9

65.1

81.4

7.0

4.7

2.3

2.3

2.3

Key: $M$, Mean; $n$, number in subsample; $S D$, standard deviation; $\%$, percentage. 
Table 2: Session content on the short training course in Cognitive and Behavioural Therapy

Session / week Content

No.

$1 . \quad$ Introduction to the course

$2 . \quad$ Introduction to principles and philosophy of CBT

3. Behavioural principles

4. Cognitions: Automatic thoughts and assumptions

5. Working with cognitions and thought diaries. Socratic methods.

6. Assessment models in CBT

7. Problems, strengths and needs list

8. Case formulation

9. Coping strategy enhancement

10. Measuring risk and conducting a risk assessment

11. Working with mood disorders and depression

12. Behavioural experiments and strategies for interventions

13. Working with anxiety disorders and phobias

$14 . \quad$ Relapse prevention

$15 . \quad$ Course evaluation 


\begin{tabular}{cccccc} 
Pre & Post & Wilcoxon \\
Signed & & \\
& $\begin{array}{c}\text { Median } \\
(I Q R)\end{array}$ & $\begin{array}{c}\text { Median } \\
(I Q R)\end{array}$ & $\begin{array}{c}\text { rank test } \\
\text { Z }=\end{array}$ & $d f$ & Sig. \\
\hline CTAS & $28(6)$ & $30(6)$ & -2.07 & 42 & .04 \\
Item 1 & $3(2)$ & $3.5(1)$ & -2.39 & 42 & .02 \\
Item 2 & $2(2)$ & $3(2)$ & -4.40 & 42 & $<.001$ \\
Item 3 & $2(0)$ & $2(0)$ & -0.54 & 42 & .59 n.s. \\
Item 4 & $4(1)$ & $4(1)$ & -0.03 & 42 & .98 n.s. \\
Item 5 & $3(2)$ & $3(3)$ & -1.91 & 42 & .06 n.s. \\
Item 6 & $4(1)$ & $4(1)$ & -1.34 & 42 & .18 n.s. \\
Item 7 & $2(1)$ & $4(1)$ & -3.61 & 42 & $<.001$ \\
Item 8 & $3(1)$ & $3(1)$ & -0.36 & 42 & .72 n.s. \\
Item 9 & $4(1)$ & $4(0)$ & -3.37 & 42 & $<.001$ \\
Item 10 & $2(2)$ & $3(1)$ & -1.86 & 42 & .06 n.s. \\
\hline
\end{tabular}

Table 3: Wilcoxon signed rank test scores on CTAS items pre- and posttraining

Key: CTAS, Cognitive Therapy Awareness Scale; df, degrees of freedom; IQR, interquartile range; ns, not significant; Sig., significant; $Z$, Wilcoxon signed-rank. 
Table 4: Spearman's rho inter-correlations between post-CTAS and competency components 712 on the CTS-R

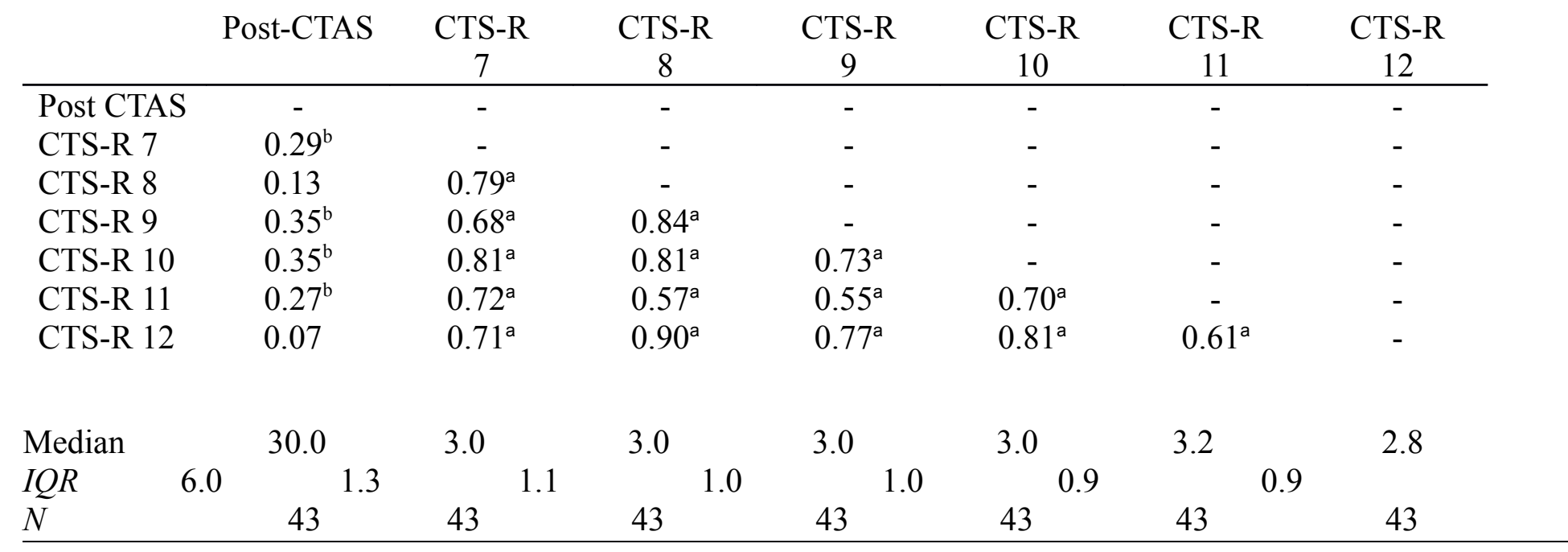

Key: $\quad$ CTAS, Cognitive Therapy Awareness Scale; CTS-R, Cognitive Therapy Scale - Revised; CTS-R 7, eliciting key cognitions; CTS-R 8, eliciting and planning behaviours;

CTS-R 9, guided discovery; CTS-R 10, conceptual integration; CTS-R 11, application of change methods; CTS-R 12, homework settings; IQR, interquartile range; $N$, total number in sample.

${ }^{\mathrm{a} C}$ Correlation is significant at the .01 level. ${ }^{\mathrm{b}}$ Correlation is significant at the .05 level. 